\title{
Domino processes as a tool to recover substandard reactions. Synthesis and use of nitroacetic acid esters and amides.
}

\author{
Noemi Scardovi, Andrea Casalini, Francesca Peri, Paolo Righi* \\ Dipartimento di Chimica Organica “A. Mangini” dell'Università \\ Viale del Risorgimento, 4 - I-40136 Bologna (Italy) \\ E-mail : righi@ms.fci.unibo.it
}

\section{Supporting Information}

General: ${ }^{1} \mathrm{H}$ NMR spectra were recorded at 300.00 or $200.00 \mathrm{MHz}$ at $20^{\circ} \mathrm{C}$ with either tetramethylsilane $(\delta 0.00)$, chloroform $(\delta 7.25)$, methanol $(\delta 3.30)$, dimethylsulfoxide $(\delta 2.49)$ or acetone $(\delta 2.20)$ as the internal standard. ${ }^{13} \mathrm{C}$ NMR spectra were recorded at 75.46 or $50.3 \mathrm{MHz}$ at $20^{\circ} \mathrm{C}$ with either chloroform $(\delta 77.0)$ methanol $(\delta 49.3)$, dimethylsulfoxide $(\delta 39.0)$ or acetone $(\delta 29.8)$ as the internal standard. Signal multiplicities were established by DEPT experiments. Melting points were determined through a Büchi instrument and are uncorrected. Specific optical rotations were determined at the sodium D line through a Perkin Elmer 341 polarimeter. Flash chromatographic separations were performed over Merck Silica gel 60 (230-400 mesh), TLC analyses were performed over Merck precoated TLC plates (Silica gel $60 \mathrm{GF}_{254} 0.25$ $\mathrm{mm}$ ). GC analyses were performed on HP 6890 instrument with a split/splitless injector, a FID detector, and a 5\% phenyl methyl silicone column $(30 \mathrm{~m}$, i.d. $0.32 \mathrm{~mm}$, film thickness $0.0025 \mathrm{~mm})$. Unless otherwise stated, solvents and reagents were used as received. Polymer-supported nitrite (Amberlyst $\mathrm{A} 26, \mathrm{NO}_{2}^{-}$form, loading ca. 4 mequiv/g wet resin) was obtained from Fluka Co. and was dried overnight in vacuum at room temperature before use (average weight loss $14 \%$ ).

Bromoacetic acid derivatives. Ethyl bromoacetate was purchased from Aldrich Chemical Co., and used as received. All other bromoacetic acid derivatives were prepared in one or two steps from the corresponding alcohol or amine and bromoacetyl bromide (Aldrich Chemical Co.). In particular, (-)-menthyl bromoacetate, (+)-4,4-dimethyl-2oxotetrahydrofuran-3-yl bromoacetate, and $t$-butyl bromoacetate were prepared by adaptation of the procedure reported by: Smiles, J. J. Chem. Soc. 1905, 87, 460; bromoacetic acid $N, N$-Diisopropyl amide and $N$-bromoacetyl proline methyl ester were prepared by adaptation of the procedure reported by: Weaver, W. E.; Whaley, W. M. J. Am. Chem. Soc. 1947, 69, 515 as modified by Hand, E. S.; Johnson, S. C.; Baker, D. C. J. Org. Chem. 1997, 62, 1348; 2-bromoacetyl-4,4-dimethyl-4,5dihydrooxazole was prepared according to Breton, P.; Andre-Barres, C.; Langlois, Y. Synth. Commun. 1992, 17, 2543.

General procedure for the halogen exchange reaction of bromoacetic acid derivatives with polymer supported nitrite. Polymer supported nitrite resin, commercially available from Fluka Co. (0.5g wet, ca. 4 mequiv per gram of wet resin, $2 \mathrm{mmol}$ ), was charged in three-necked $25-\mathrm{mL}$ reaction flask equipped with a thermometer, a septum, and a nitrogen inlet and was dried overnight in vacuum at room temperature (average weight loss: $14 \%)$. Acetonitrile $(5 \mathrm{~mL})$ was added to resin and the suspension was stirred at room temperature for $30 \mathrm{~min}$. The mixture was cooled to ca. $-15^{\circ} \mathrm{C}$ with the aid of a finely crushed $3: 1$ ice-salt bath. The bromoacetic acid derivative $(1 \mathrm{mmol})$ was then added via syringe through a septum. The reaction was monitored by GC until disappearance of the starting material. The mixture was quickly filtered while still cold through a glass funnel and the resin was washed several times with small portions of methylene chloride. Pure samples were obtained by flash column chromatography (ethyl acetate/pet ether). 


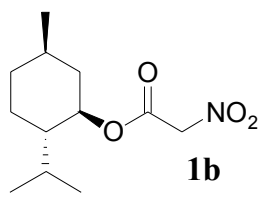

$(1 R, 2 S, 5 R)$-2-Isopropyl-5-methylcyclohex-1-yl nitroacetate [(-)-menthyl nitroacetate] (1b): ${ }^{1} \mathrm{H}$ NMR $\left(\mathrm{CDCl}_{3}\right) \delta 5.15(s, 2 \mathrm{H}), 4.80(d t, 1 \mathrm{H}, J=11.0, J=4.8) ; 2.10-1.95(m, 1 \mathrm{H}) ; 1.90-0.70(m, 8 \mathrm{H})$; $0.89(d, 3 \mathrm{H}, J=4.4) ; 0.86(d, 3 \mathrm{H}, J=5.1) ; 0.74(d, 3 \mathrm{H}, J=7.0) \mathrm{ppm} .{ }^{13} \mathrm{C} \mathrm{NMR}\left(\mathrm{CDCl}_{3}\right) \delta 161.30(s)$; $76.45(d) ; 76.36(t) ; 46.70(d) ; 40.31(t) ; 33.89(t) ; 31.39(d) ; 26.10(d) ; 23.18(t) ; 21.82(q) ; 20.86(q)$; $16.05(q)$ ppm. $\mathrm{R}_{f} 0.36(\mathrm{EA}: \mathrm{PE}=9: 1) . \mathrm{GC} 8.8 \mathrm{~min}\left(100^{\circ} \mathrm{C}, 2 \mathrm{~min}\right.$ then $\left.15{ }^{\circ} \mathrm{C} / \mathrm{min}\right) \cdot[\alpha]^{20}{ }_{\mathrm{D}}-67.1(c$

$\left.0.85, \mathrm{CHCl}_{3}\right)$.

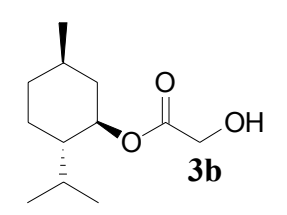

$(1 R, 2 S, 5 R)$-2-Isopropyl-5-methylcyclohex-1-yl hydroxyacetate [(-)-menthyl hydroxyacetate] (3b): ${ }^{1} \mathrm{H}$ NMR $\left(\mathrm{CDCl}_{3}\right) \delta 4.81(t d, 1 \mathrm{H}, J=10.7, J=4.4) ; 4.15(s, 2 \mathrm{H}) ; 2.80(b s, 1 \mathrm{H}) ; 2.10-1.95(m, 1 \mathrm{H})$; $1.90-0.70(m, 8 \mathrm{H}) ; 0.92(d, 3 \mathrm{H}, J=6.3) ; 0.89(d, 3 \mathrm{H}, J=7.1) ; 0.76(d, 3 \mathrm{H}, J=7.1) \mathrm{ppm} .{ }^{13} \mathrm{C}$ NMR $\left(\mathrm{CDCl}_{3}\right) \delta 172.96(s) ; 75.69(d) ; 60.56(t) ; 46.87(d) ; 40.71(t) ; 34.01(t) ; 31.29(d) ; 26.20(d) ; 23.32(t)$; $21.88(q) ; 20.60(q) ; 16.21(q)$ ppm. $\mathrm{R}_{f} 0.22(\mathrm{EA}: \mathrm{PE}=9: 1)$. GC $7.5 \mathrm{~min}\left(100^{\circ} \mathrm{C}, 2 \mathrm{~min}\right.$ then $\left.15^{\circ} \mathrm{C} / \mathrm{min}\right) \cdot[\alpha]_{\mathrm{D}}^{20}-77.5\left(\mathrm{c} 1.00, \mathrm{CHCl}_{3}\right)$.

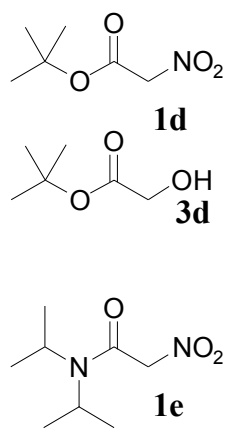

tert-Butyl nitroacetate (1d): ${ }^{1} \mathrm{H}$ NMR $\left(\mathrm{CDCl}_{3}\right) \delta 5.12(s, 2 \mathrm{H}) ; 1.51(s, 9 \mathrm{H}) \mathrm{ppm} .{ }^{13} \mathrm{C} \mathrm{NMR}\left(\mathrm{CDCl}_{3}\right) \delta$ $160.89(s) ; 84.91(s) ; 77.11(t) ; 27.74(q) \mathrm{ppm}$. GC $6.7 \mathrm{~min}\left(60{ }^{\circ} \mathrm{C}, 2 \mathrm{~min}\right.$ then $\left.15^{\circ} \mathrm{C} / \mathrm{min}\right)$.

tert-Butyl hydroxyacetate (3d): ${ }^{1} \mathrm{H}$ NMR $\left(\mathrm{CDCl}_{3}\right) \delta 4.0(s, 2 \mathrm{H}) ; 2.9(s, 1 \mathrm{H}) 1.48(s, 9 \mathrm{H}) \mathrm{ppm} .{ }^{13} \mathrm{C}$ NMR $\left(\mathrm{CDCl}_{3}\right) \delta 172.49(s) ; 82.15(s) ; 60.81(t) ; 27.61(q)$ ppm. GC $3.5 \min \left(60^{\circ} \mathrm{C}, 2 \mathrm{~min}\right.$ then $\left.15^{\circ} \mathrm{C} / \mathrm{min}\right)$.

$N, N$-Diisopropyl nitroacetamide (1e): ${ }^{1} \mathrm{H}$ NMR $\left(\mathrm{CDCl}_{3}\right) \delta 5.30(s, 2 \mathrm{H}) ; 3.40-3.70(m, 1 \mathrm{H}$, cis and trans $\left.\mathrm{CH}\left(\mathrm{CH}_{3}\right)_{2}\right) ; 1.41\left(d, J=6.8\right.$, cis $\left.\left(\mathrm{CH}_{3}\right)_{2} \mathrm{CH}\right) ; 1.26\left(d, J=6.6\right.$, trans $\left.\left(\mathrm{CH}_{3}\right)_{2} \mathrm{CH}\right) \mathrm{ppm} .{ }^{13} \mathrm{C} \mathrm{NMR}$ $\left(\mathrm{CDCl}_{3}\right) \delta 170.29(s) ; 79.17(t) ; 49.87\left(d\right.$, cis $\left.\mathrm{CH}\left(\mathrm{CH}_{3}\right)_{2}\right) ; 47.27\left(d\right.$, trans $\left.\mathrm{CH}\left(\mathrm{CH}_{3}\right)_{2}\right) ; 21.13$ (q, cis $\left.\left(\mathrm{CH}_{3}\right)_{2} \mathrm{CH}\right) ; 20.60\left(q\right.$, trans $\left.\left(\mathrm{CH}_{3}\right)_{2} \mathrm{CH}\right) \mathrm{ppm}$. GC $8.3 \mathrm{~min}\left(80^{\circ} \mathrm{C}, 2 \mathrm{~min}\right.$ then $\left.15^{\circ} \mathrm{C} / \mathrm{min}\right)$.

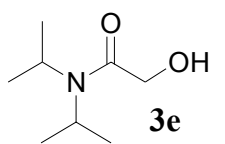

$N, N$-Diisopropyl hydroxyacetamide (3e): ${ }^{1} \mathrm{H}$ NMR $\left(\mathrm{CDCl}_{3}\right) \delta 4.10(s, 2 \mathrm{H}) ; 3.75-3.40(m, 1 \mathrm{H}$, cis and trans $\left.\mathrm{CH}\left(\mathrm{CH}_{3}\right)_{2}\right) ; 1.42\left(d, J=6.8\right.$, cis $\left.\left(\mathrm{CH}_{3}\right)_{2} \mathrm{CH}\right) ; 1.21\left(d, J=6.7\right.$, trans $\left.\left(\mathrm{CH}_{3}\right)_{2} \mathrm{CH}\right) \mathrm{ppm} .{ }^{13} \mathrm{C}$ NMR $\left(\mathrm{CDCl}_{3}\right) \delta 159.7(s) ; 60.70(t) ; 47.27\left(d\right.$, cis $\left.\mathrm{CH}\left(\mathrm{CH}_{3}\right)_{2}\right)$; 46.52 (d) trans $\left.\mathrm{CH}\left(\mathrm{CH}_{3}\right)_{2}\right)$; 20.94 (q, cis $\left.\left(\mathrm{CH}_{3}\right)_{2} \mathrm{CH}\right) ; 20.85$ (q, trans $\left.\left(\mathrm{CH}_{3}\right)_{2} \mathrm{CH}\right) \mathrm{ppm}$. GC $6.5 \min \left(80^{\circ} \mathrm{C}, 2 \mathrm{~min}\right.$ then $\left.15^{\circ} \mathrm{C} / \mathrm{min}\right) . \mathrm{R}_{\mathrm{f}} 0.23(\mathrm{EA}: \mathrm{PE}=$

$4: 1)$.

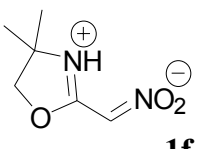

4,4-Dimethyl-2-nitromethyl-4,5-dihydrooxazole (1f): ${ }^{1} \mathrm{H}$ NMR $\left(\mathrm{CDCl}_{3}\right) \delta 1.53(s, 6 \mathrm{H}) ; 4.32(s, 2 \mathrm{H})$; $5.95(s, 1 \mathrm{H}) ; 6.57(\mathrm{~s}, 1 \mathrm{H}) \mathrm{ppm} .{ }^{13} \mathrm{C}$ NMR $\left(\mathrm{CDCl}_{3}\right) \delta 25.12(q) ; 58.15(s) ; 78.71(t) ; 93.29(d) ; 96.18(s)$; $157.43(s) \mathrm{ppm}$. MS (EI, $70 \mathrm{eV}) 158(1) \mathrm{M}^{+} ; 143(24) \mathrm{M}^{+}-\mathrm{CH}_{3} ; 109(20) \mathrm{M}^{+}-\mathrm{NO}_{2} ; 82(60) ; 42$ (100)

1f $\mathrm{CH}_{2} \mathrm{OC}^{+} \mathrm{m} / \mathrm{z}$.

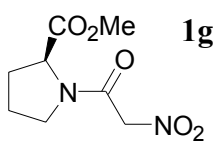

$N$-Nitroacetyl proline methyl ester (1g): ${ }^{1} \mathrm{H}$ NMR $\left(\mathrm{CD}_{3} \mathrm{OD}\right) \delta 5.29\left(d, J=13.7, \mathrm{CH}_{\mathrm{a}} \mathrm{NO}_{2} ;\right.$ cis); $5.23(d$, $J=13.7, \mathrm{CH}_{\mathrm{b}} \mathrm{NO}_{2}$ cis); 5.21 ( $d, J=13.5, \mathrm{CH}_{\mathrm{a}} \mathrm{NO}_{2}$ trans); 5.09 ( $d, J=13.5, \mathrm{CH}_{\mathrm{b}} \mathrm{NO}_{2}$ trans); 4.52-4.44 ( $m$, $\mathrm{C}^{2} \mathrm{H}$ cis); 4.43-4.35 ( $m, \mathrm{C}^{2} \mathrm{H}$, trans); 3.90-3.30 ( $m, \mathrm{C}^{5} \mathrm{H}_{2}$ cis and trans); $3.72\left(s, \mathrm{CH}_{3}\right.$ trans); $3.65\left(s, \mathrm{CH}_{3}\right.$ cis); 2.30-1.70 ( $m, \mathrm{C}^{3} \mathrm{H}_{2}$ and $\mathrm{C}^{4} \mathrm{H}_{2}$, cis and trans) ppm. ${ }^{13} \mathrm{C}$ NMR $\left(\mathrm{CD}_{3} \mathrm{OD}\right) \delta 172.25(s) ; 160.34(s) ; 77.99$ ( $t, \mathrm{CH}_{2} \mathrm{NO}_{2}$ cis and trans); $59.92\left(d, \mathrm{C}^{2} \mathrm{H}\right.$ trans); 59.82 ( $d, \mathrm{C}^{2} \mathrm{H}$ cis); $53.64\left(q, \mathrm{CH}_{3} \operatorname{trans}\right) ; 52.99\left(q, \mathrm{CH}_{3}\right.$ cis); $47.75\left(t, \mathrm{C}^{5} \mathrm{H}_{2} \mathrm{~N}\right.$ trans); $47.43\left(t, \mathrm{C}^{5} \mathrm{H}_{2} \mathrm{~N}\right.$ cis); $31.49\left(t, \mathrm{C}^{3} \mathrm{H}_{2}\right.$ trans); $29.60\left(t, \mathrm{C}^{3} \mathrm{H}_{2}\right.$ cis $) ; 25.15\left(t, \mathrm{C}^{4} \mathrm{H}_{2}\right.$ cis $) ; 22.93\left(t, \mathrm{C}^{4} \mathrm{H}_{2}\right.$ trans $) \mathrm{ppm}$. MS (EI) $\left.186\left([\mathrm{M}-\mathrm{NO}]^{+}\right) ; 170\left(11 \%,\left[\mathrm{M}-\mathrm{NO}_{2}\right]^{+}\right) ; 128\left(10 \%,\left[\mathrm{M}-\mathrm{COCH}_{2} \mathrm{NO}_{2}\right]^{+}\right) ; 111\left(38 \% \text {, [M-COOMe }-\mathrm{NO}_{2}\right]^{+}\right) ; 83(100 \%$, $\left.\left[\mathrm{C}_{5} \mathrm{H}_{9} \mathrm{~N}\right]^{+}\right) ; 70\left(44 \%,\left[\mathrm{C}_{4} \mathrm{H}_{8} \mathrm{~N}\right]^{+} \mathrm{m} / \mathrm{z}\right.$. GC $11.4 \min \left(80{ }^{\circ} \mathrm{C}, 2 \min\right.$ then $\left.15{ }^{\circ} \mathrm{C} / \mathrm{min}\right)$.

General preparation of 4-hydroxy-4,5-dihydroisoxazoles 2-oxides of type 5 through the domino process. To a stirred solution of the epoxy alcohol $(2 \mathrm{mmol})$ in acetonitrile $(5.0 \mathrm{~mL})$ 2,2,6,6-tetramethyl-1-piperidinyloxyl (TEMPO) ( $0.2 \mathrm{mmol} ; 0.1$ equiv) and bisacetoxyiodobenzene (BAIB, $2.2 \mathrm{mmol})$ were successively added at room temperature. The course of the reaction was monitored by tlc (ethyl acetate/hexane $=40 / 60)$ until the alcohol was consumed $(3-5 \mathrm{~h})$. At this point the mixture was cooled to ca. $0^{\circ} \mathrm{C}$ with the aid of a crushed ice/water bath. Then diisopropylethyl amine (DIPEA, $6.6 \mathrm{mmol})$, the bromoacetic acid derivative $(2 \mathrm{mmol})$, and dried polymer-supported nitrite $(4.0 \mathrm{~g}$ wet, $4.0 \mathrm{mmol})$ were subsequently added. The reaction mixture was set aside in a refrigerator $\left(0{ }^{\circ} \mathrm{C} \div 2{ }^{\circ} \mathrm{C}\right)$ overnight. The reaction mixture was quickly filtered over a glass filter and the solids were carefully washed with several portions of dichloromethane. The organic layer was concentrated in vacuum, and the crude product was purified by flash chromatography. 
4,5-Dihydroisoxazoles (-)-4,5-trans-5b and (-)-4,5-cis-5b. The reaction was performed using (2S,3S)-trans3-phenylglycidol as the epoxy alcohol and $l-(-)$-menthyl bromoacetate. The two diastereoisomer were separated by flash chromatography (dichloromethane : diethyl ether $=92: 8$ ) obtaining $380 \mathrm{mg}$ of 4,5-cis-5b and $253 \mathrm{mg}$ of 4,5-trans-5b (clear oil, $633 \mathrm{mg}$ combined, $81 \%$ yield).

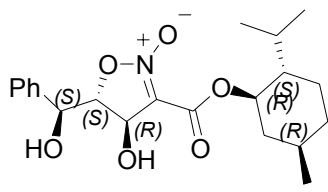

(-)-4,5-trans-5b. ${ }^{1} \mathrm{H}$ NMR $\left(\mathrm{CDCl}_{3}\right) \delta 0.75(d, 3 \mathrm{H}, J=6.7) ; 0.88(d, 3 \mathrm{H}, J=7.1) ; 0.92(d, 3 \mathrm{H}$, $J=6.6) ; 1.01-1.15(m, 2 \mathrm{H}) ; 1.22-1.34(m, 1 \mathrm{H}) ; 1.41-1.58(m, 2 \mathrm{H}) ; 1.63-1.77(m, 2 \mathrm{H}) ; 1.78-1.91$ $(m, 1 \mathrm{H}) ; 1.98-2.10(m, 1 \mathrm{H}) ; 2.73(b s, 1 \mathrm{H}) ; 2.78(b d, 1 \mathrm{H}, J=3.6) ; 4.67(d d, 1 \mathrm{H}, J=2.8, J=4.2)$; $4.88(d t, 1 \mathrm{H}, J=4.4, J=10.9) ; 5.05(b s, 1 \mathrm{H}) ; 5.53(b s, 1 \mathrm{H}) ; 7.32-7.47(m, 5 \mathrm{H}) \mathrm{ppm} .{ }^{13} \mathrm{C} \mathrm{NMR}$ $\left(\mathrm{CDCl}_{3}\right) \delta 16.09(q) ; 20.80(q) ; 21.93(q) ; 23.18(t) ; 26.12(d) ; 31.43(d) ; 34.00(t) ; 40.75(t) ; 46.81$ $(d) ; 71.95(d) ; 73.30(d) ; 76.47(d) ; 85.46(d) ; 110.73(s) ; 126.16(d) ; 128.62(d) ; 128.83(d) ; 137.13(s) ; 158.52(s)$ ppm. $\mathrm{R}_{\mathrm{f}}$ $0.18\left(\mathrm{CH}_{2} \mathrm{Cl}_{2}: \mathrm{Et}_{2} \mathrm{O}=92: 8\right)$. IR $(\mathrm{KBr}) \vee 3464,2959,2929,2871,1711,1617,1242,1041 \mathrm{~cm}^{-1}$. MS (EI, $\left.70 \mathrm{eV}\right) 360\left(\mathrm{M}^{+}\right.$31); 195, 139, 107, $83(100), 69,55 \mathrm{~m} / z .[\alpha]_{\mathrm{D}}{ }^{23}-79.2\left(c 0.795, \mathrm{CHCl}_{3}\right)$. Mp. 122-126 ${ }^{\circ} \mathrm{C}$.Anal. Calcd for $\mathrm{C}_{21} \mathrm{H}_{29} \mathrm{NO}_{6}: \mathrm{C}_{\text {, }}$ 64.43; H, 7.47; N, 3.58. Found: C, 64.31; H, 7.46; N, 3.53.

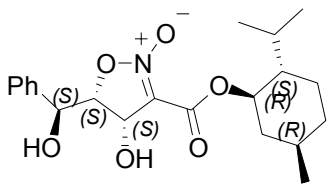

(-)-4,5-cis-5b. ${ }^{1} \mathrm{H}$ NMR $\left(\mathrm{CDCl}_{3}\right) \delta 0.76(d, 3 \mathrm{H}, J=7.1) ; 0.91(d, 3 \mathrm{H}, J=7.1) ; 0.92(d, 3 \mathrm{H}, J=$ $6.3) ; 1.02-1.18(m, 2 \mathrm{H}) ; 1.22-1.38(m, 1 \mathrm{H}) ; 1.41-1.58(\mathrm{~m}, 2 \mathrm{H}) ; 1.65-1.77(\mathrm{~m}, 2 \mathrm{H}) ; 1.85-1.96(\mathrm{~m}$, $1 \mathrm{H}) ; 2.01-2.12(m, 1 \mathrm{H}) ; 3.30(b s, 1 \mathrm{H}) ; 3.59(b s, 1 \mathrm{H}) ; 4.67(d d, 1 \mathrm{H}, J=6.4, J=8.1) ; 4.90(d t, 1 \mathrm{H}$, $J=4.6, J=10.8) ; 5.22(b d, 1 \mathrm{H}, J=8.0) ; 5.55(b d, 1 \mathrm{H}, J=6.3) ; 7.32-7.47(m, 5 \mathrm{H}) \mathrm{ppm} .{ }^{13} \mathrm{C}$ NMR $\left(\mathrm{CDCl}_{3}\right) \delta 16.21(q) ; 20.77(q) ; 21.93(q) ; 23.27(t) ; 26.19(s) ; 31.43(s) ; 34.02(t) ; 40.65(t) ; 46.83$ $(d) ; 70.32(d) ; 73.36(d) ; 76.58(d) ; 80.93(d) ; 111.26(s) ; 126.62(d) ; 128.67(d) ; 128.70(d) ; 138.83(s) ; 158.60(s)$ ppm. $\mathrm{R}_{\mathrm{f}}$ $0.29\left(\mathrm{CH}_{2} \mathrm{Cl}_{2}: \mathrm{Et}_{2} \mathrm{O}=92: 8\right)$. IR (KBr) $\vee 3464,2958,2928,2872,1723,1617,1241,1019 \mathrm{~cm}^{-1} .[\alpha]_{\mathrm{D}}{ }^{20}-41.2(c 0.868$, $\mathrm{CHCl}_{3}$ ). $\mathrm{Mp} 48-51{ }^{\circ} \mathrm{C}$. Anal. Calcd for $\mathrm{C}_{21} \mathrm{H}_{29} \mathrm{NO}_{6}: \mathrm{C}, 64.43 ; \mathrm{H}, 7.47 ; \mathrm{N}, 3.58$. Found: C, 64.50; H, 7.44; N, 3.59 .

4,5-Dihydroisoxazoles $( \pm)-4,5-t r a n s-5 e$ and $( \pm)-4,5-c i s-5$. The reaction was performed using racemic trans-3phenylglycidol as the epoxy alcohol and $N, N$-diisopropyl bromoacetamide. The two diastereoisomer were separated by flash chromatography (ethyl acetate : pet ether $=50: 50$ ) obtaining $274 \mathrm{mg}$ of 4,5-cis-5e and $224 \mathrm{mg}$ of 4,5-trans-5e (498 $\mathrm{mg}$ combined, $74 \%$ yield). The isolated isomers were found to be only sparingly soluble in any deuterated solvent. Acceptable spectra were obtained in acetone- $d_{6}$. Better spectra were obtained for the corresponding bis- $O$-(tert-butyldimethylsilyl) ethers prepared according to a procedure described elsewhere. ${ }^{1}$

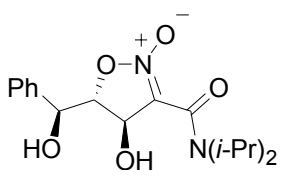

( \pm )-4,5-trans-5e. ${ }^{1} \mathrm{H}$ NMR (acetone- $\left.d_{6}\right) \delta$ 1.20-1.50 (m, 12H); $3.60(m, 1 \mathrm{H}) ; 3.90(m, 1 \mathrm{H}) ; 4.58(d d$, $\mathrm{J}=4.8, \mathrm{~J}=2.4) ; 4.98(d, 1 \mathrm{H}, \mathrm{J}=4.8) ; 5.10-5.40(b s, 2 \mathrm{H}) ; 5.46(d, 1 \mathrm{H}, \mathrm{J}=2.4) ; 7.20-7.60(m, 5 \mathrm{H})$ ppm. ${ }^{13} \mathrm{C}$ NMR (acetone- $\left.d_{6}\right) \delta 20.77(q), 21.54(q), 21.80(q) ; 47.16(d), 51.82(d) ; 73.04(d), 76.13(d)$ , $88.51(d) ; 114.44(s) ; 127.9(d), 128.9(d), 129.5(d) ; 141.09(s) ; 159.4(s)$ ppm. $\mathrm{R}_{\mathrm{f}} 0.29(\mathrm{EA}: \mathrm{PE}=$ $1: 1)$. IR $(\mathrm{KBr}) \vee 3363,3172,2973,2938,1654,1617,1031 \mathrm{~cm}^{-1}$. Mp $155-157^{\circ} \mathrm{C}$.

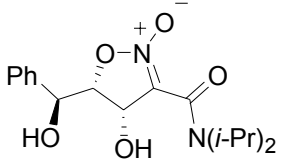

$( \pm)-4,5$-cis-5e. ${ }^{1} \mathrm{H}$ NMR (acetone- $\left.d_{6}\right) \delta 1.20-1.50(m, 12 \mathrm{H}) ; 3.60(m, 1 \mathrm{H}) ; 3.90(m, 1 \mathrm{H}) ; 4.70(d d$, $J=8.6, J=6.0)$; $5.15(d, 1 \mathrm{H}, J=8.7) ; 4.8-5.4(b s, 2 \mathrm{H}) ; 5.45(d, 1 \mathrm{H}, J=6.1) ; 7.20-7.50(m, 5 \mathrm{H}) \mathrm{ppm}$. ${ }^{13} \mathrm{C}$ NMR (acetone- $\left.d_{6}\right) \delta 20.72(q), 21.34(q), 21.82(q) ; 47.21(d), 52.05(d) ; 70.39(d), 75.42(d)$, $84.03(d) ; 115.61(\mathrm{~s}) ; 128.43(d), 129.06(d), 129.26(d) ; 142.64(s) ; 159.49(s)$ ppm. $\mathrm{R}_{\mathrm{f}} 0.23(\mathrm{EA}:$ $\mathrm{PE}=1: 1) . \mathrm{IR}(\mathrm{KBr}) \vee 3313,2973,2934,1637,1608,1021 \mathrm{~cm}^{-1}$. Mp $157-159^{\circ} \mathrm{C}$

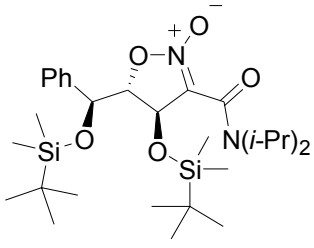

( \pm )-Bis-O-(tert-butyldimethylsilyl)-4,5-trans-5e. ${ }^{1} \mathrm{H}$ NMR $\left(\mathrm{CDCl}_{3}\right) \delta-0.54(s, 3 \mathrm{H}),-0.19(s, 3 \mathrm{H})$, $-0.07(s, 3 \mathrm{H}), 0.15(s, 3 \mathrm{H}) ; 0.67(s, 9 \mathrm{H}), 0.90(s, 9 \mathrm{H}) ; 1.17(d, 3 \mathrm{H}, J=6.7) ; 1.34(d, 3 \mathrm{H}, J=6.3)$; $1.41(d, 3 \mathrm{H}, J=6.8) ; 1.46(d, 3 \mathrm{H}, J=6.8) ; 3.48$ (hept, $1 \mathrm{H}, J=6.9) ; 3.90$ (hept, $1 \mathrm{H}, J=6.4) ; 4.37$ $(d d, 1 \mathrm{H}, J=1.5, J=2.8) ; 5.07(d, 1 \mathrm{H}, J=2.7) ; 5.60(d, 1 \mathrm{H}, J=1.5) ; 7.20-7.50(m, 5 \mathrm{H}) \mathrm{ppm} .{ }^{13} \mathrm{C}$ $\operatorname{NMR}\left(\mathrm{CDCl}_{3}\right) \delta-5.67(q),-4.72(q),-4.55(q),-4.29(q), 18.08(s), 18.54(s) ; 20.17(q), 20.69(q)$, 21.09 (q), $22.33(q) ; 25.92(q), 26.14(q) ; 46.98(d), 50.97(d) ; 74.43(d) ; 75.45(d) ; 88.71(d)$; $114.93(s) ; 126.97(d), 128.56(d), 129.16(d) ; 138.68(s) ; 157.70(s)$ ppm. ${\mathrm{Mp} 124^{\circ} \mathrm{C}-125^{\circ} \mathrm{C} \mathrm{R} f}_{f} 0.36\left(\mathrm{PE}: \mathrm{Et}_{2} \mathrm{O}=9: 1\right)$. IR $(\mathrm{KBr}) \vee 2961,2932,2859,1635,1259,1086 \mathrm{~cm}^{-1}$. Anal calcd for $\mathrm{C}_{29} \mathrm{H}_{52} \mathrm{~N}_{2} \mathrm{O}_{5} \mathrm{Si}_{2}: \mathrm{C}, 61.66 ; \mathrm{H}, 9.28 ; \mathrm{N}, 4.96$. Found: C, $61.39 ; \mathrm{H}, 9.32 ; \mathrm{N}, 5.01$.

\footnotetext{
${ }^{1}$ Marotta, E.; Micheloni, L. M.; Scardovi, N.; Righi, P. Org. Lett. 2001, 3, 727.
} 


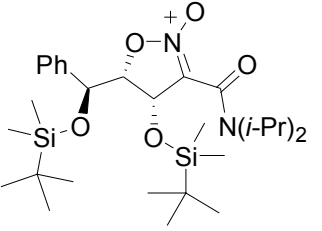

( \pm )-Bis- $O$-(tert-butyldimethylsilyl)-4,5-cis-5e. ${ }^{1} \mathrm{H}$ NMR $\left(\mathrm{CDCl}_{3}\right) \delta-0.25(s, 3 \mathrm{H}),-0.03(s, 3 \mathrm{H})$, $0.16(s, 3 \mathrm{H}), 0.25(s, 3 \mathrm{H}) ; 0.76(d, 3 \mathrm{H}, J=6.5), 0.83(s, 9 \mathrm{H}) ; 0.87(d, 3 \mathrm{H}, J=6.6) ; 1.00(s, 9 \mathrm{H})$; $1.23(d, 3 \mathrm{H}, J=6.7) ; 1.26(d, 3 \mathrm{H}, J=6.7), 2.14$ (hept, $1 \mathrm{H}, J=6.7), 3.28$ (hept, $1 \mathrm{H}, J=6.7$ ); 5.00 $(d d, 1 \mathrm{H}, J=1.5, J=9.3) ; 5.15(d, 1 \mathrm{H}, J=1.5) ; 5.95(d, 1 \mathrm{H}, \mathrm{J}=9.3) ; 7.20-7.50(m, 3 \mathrm{H}) ; 7.65(d d$, $2 \mathrm{H}, J=1.6, J=7.8) \mathrm{ppm} .{ }^{13} \mathrm{C}$ NMR $\left(\mathrm{CDCl}_{3}\right) \delta-4.63(q),-4.48(q),-4.39(q),-4.20(q) ; 18.47(s)$, $18.74(s) ; 20.08(q), 20.71(q), 20.76(q) ; 22.71(q) ; 26.13(q), 26.38(q) ; 46.73(d), 50.77(d) ; 74.09$ $(d) ; 76.14(d) ; 84.69(d) ; 112.81(s) ; 128.15(d), 129.70(d) ; 139.25(s) ; 157.99(s)$ ppm. Mp $124^{\circ} \mathrm{C}-126^{\circ} \mathrm{C} \mathrm{R} f 0.14\left(\mathrm{PE}: \mathrm{Et}_{2} \mathrm{O}\right.$ $=9: 1)$. IR $(\mathrm{KBr}) \vee 2959,2930,2859,1635,1260,1091 \mathrm{~cm}^{-1}$. Anal calcd for $\mathrm{C}_{29} \mathrm{H}_{52} \mathrm{~N}_{2} \mathrm{O}_{5} \mathrm{Si}_{2}: \mathrm{C}, 61.66 ; \mathrm{H}, 9.28 ; \mathrm{N}, 4 ., 96$. Found: C, 61.,78; H, 9.29; N, 4.93.

4,5-Dihydroisoxazoles $( \pm)-4,5-t r a n s-5 f$ and $( \pm)-4,5$-cis-5f. The reaction was performed using racemic glycidol as the epoxy alcohol and 2-(bromomethyl)-4,4-dimethyl-4,5-dihydrooxazole. The two diastereoisomer were separated by flash chromatography (dichloromethane : methanol = 94:6) obtaining $246 \mathrm{mg}$ of 4,5-cis-5f and $164 \mathrm{mg}$ of 4,5-trans-5f (410 $\mathrm{mg}$ combined, $89 \%$ yield). The isolated isomers were found to be only sparingly soluble in any deuterated solvent. Acceptable spectra were obtained in acetone- $d_{6}$. Better spectra were obtained for the corresponding bis- $O$-(tert-butyldimethylsilyl) ethers prepared as described elsewhere. ${ }^{1}$
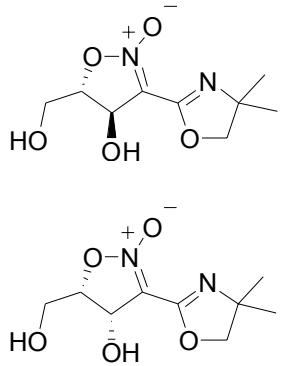

$( \pm)-4,5$-trans-5f. ${ }^{1} \mathrm{H}$ NMR (acetone- $\left.d_{6}\right) \delta 1.27(s, 6 \mathrm{H}) ; 3.63(d d, 1 \mathrm{H}, J=11.8, J=5.8) ; 3.74(d d$, $1 \mathrm{H}, J=11.8, J=4.7) ; 4.00(s, 2 \mathrm{H}) ; 4.57(d d d, 1 \mathrm{H}, J=2.0, J=5.8, J=4.2) ; 5.50(d, 1 \mathrm{H}, J=2.0)$ ppm. ${ }^{13} \mathrm{C}$ NMR (acetone- $\left.d_{6}\right) \delta 24.32(q ;) 61.15(t) ; 68.30(s) ; 75.39(t) ; 75.39(d) ; 85.90(d) ; 109.83$ $(s) ; 154.58(s)$ ppm.

$( \pm)-4,5$-cis-5f. ${ }^{1} \mathrm{H}$ NMR (acetone- $\left.d_{6}\right) \quad \delta 1.29(s, 6 \mathrm{H}) ; 3.78(d d, 1 \mathrm{H}, J=11.7, J=6.3) ; 4.04(s, 2 \mathrm{H})$; $4.68(d d d, 1 \mathrm{H}, J=5.5, J=6.3, J=5.8) ; 5.58(d, 1 \mathrm{H}, \mathrm{J}=5.8) \mathrm{ppm} .{ }^{13} \mathrm{C}$ NMR (acetone- $\left.d_{6}\right) \delta 28.08(q)$; $58.80(t) ; 67.39(s) 73.62(t) ; 79.55(d) ; 83.77(d) ; 112.30(s) ; 154.55(s)$ ppm.

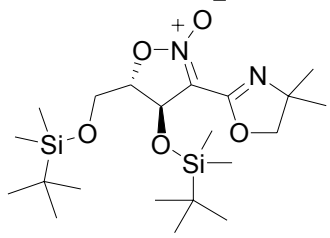

( \pm )-Bis-O-(tert-butyldimethylsilyl)-4,5-trans-5f. ${ }^{1} \mathrm{H}$ NMR $\left(\mathrm{CDCl}_{3}\right) \delta 0.08(s, 6 \mathrm{H}), 0.14(s, 3 \mathrm{H})$, $0.16(s, 3 \mathrm{H}), 0.85(s, 9 \mathrm{H}), 0.87(s, 9 \mathrm{H}), 1.33(s, 6 \mathrm{H}), 3.70(d d, 1 \mathrm{H}, J=5.1, J=11.3), 3.76(d d, 1 \mathrm{H}$, $J=4.0, J=11.3), 4.07(s, 1 \mathrm{H}), 4.08(s, 1 \mathrm{H}), 4.38(d d d, 1 \mathrm{H}, J=1.1, J=4.0, J=5.1), 5.47(d, 1 \mathrm{H}$, $J=1.1)$ ppm. ${ }^{13} \mathrm{C}$ NMR $\left(\mathrm{CDCl}_{3}\right) \delta-5.11(q) ;-4.33(q) ;-4.27(q) ; 18.45(s) ; 18.59(s) ; 25.06(q)$; $26.20(q) ; 28.75(q) ; 62.37(t) ; 68.60(s) ; 76.90(d) ; 79.38(t) ; 84.68(d) ; 109.60(s) ; 153.65(s)$ ppm. Anal calcd for $\mathrm{C}_{21} \mathrm{H}_{42} \mathrm{~N}_{2} \mathrm{O}_{5} \mathrm{Si}_{2}$ : C, 54.98; H, 9.23; N, 6.11. Found: C, 55.12; H, 9.31; N, 6.19.

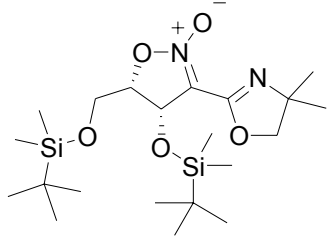

$( \pm)$-Bis- $O$-(tert-butyldimethylsilyl)-4,5-cis-5f. ${ }^{1} \mathrm{H}$ NMR $\left(\mathrm{CDCl}_{3}\right) \delta 0.09(s, 6 \mathrm{H}), 0.11(s, 3 \mathrm{H})$, $0.16(s, 3 \mathrm{H}), 0.88(s, 9 \mathrm{H}), 0.90(s, 9 \mathrm{H}), 1.34(s, 3 \mathrm{H}), 1.35(s, 3 \mathrm{H}), 3.95(d d, 1 \mathrm{H}, J=6.6, J=11.3)$, $4.04(s, 2 \mathrm{H}), 4.04(d d, 1 \mathrm{H}, J=5.2, \mathrm{~J}=11.3), 4.55(d t, 1 \mathrm{H}, J=5.2, J=6.6), 5.33(d, 1 \mathrm{H}, J=5.2)$ ppm. ${ }^{13} \mathrm{C}$ NMR $\left(\mathrm{CDCl}_{3}\right) \delta-5.46(q),-5.49(q),-5.12(q),-4.23(q) ; 18.52(s) ; 25.99(q) ; 26.07(q)$; $28.30(q) 28.51(q) ; 59.52(t) ; 68.47(s) ; 74.31(t) ; 79.24(d) ; 82.56(d) ; 111.00(s) ; 153.35(s)$ ppm. Anal calcd for $\mathrm{C}_{21} \mathrm{H}_{42} \mathrm{~N}_{2} \mathrm{O}_{5} \mathrm{Si}_{2}:$ C, 54.98; H, 9.23; N, 6.11. Found: C, 55.13; H, 9.25; N, 6.01. 


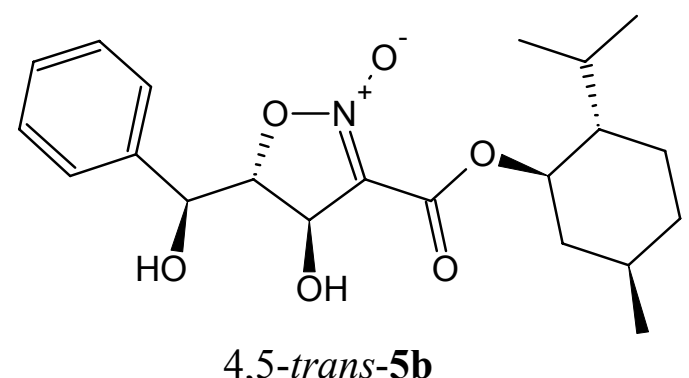

4,5-trans-5b

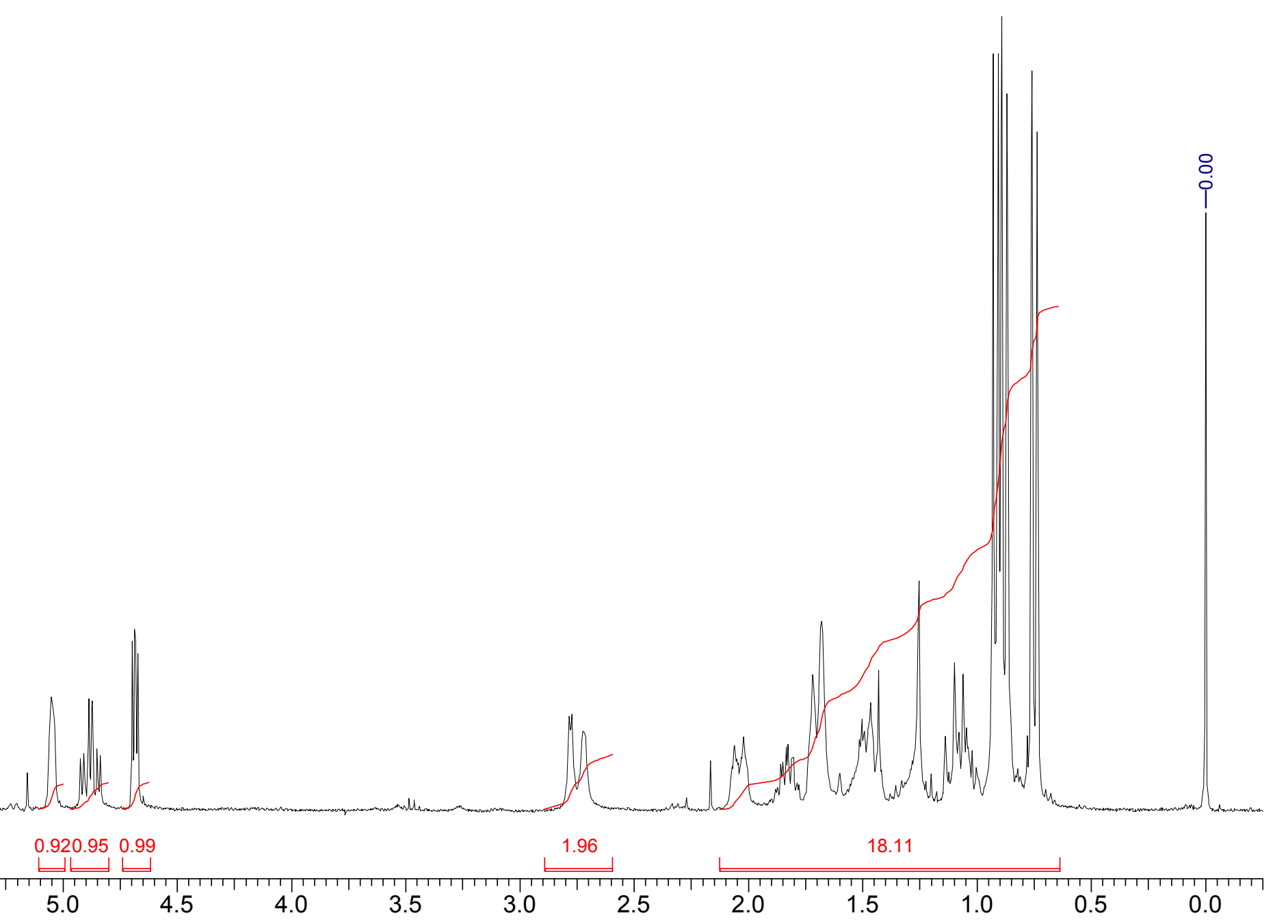




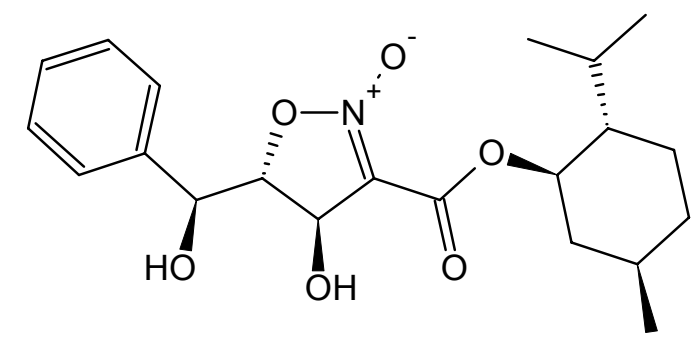

4,5-trans-5b

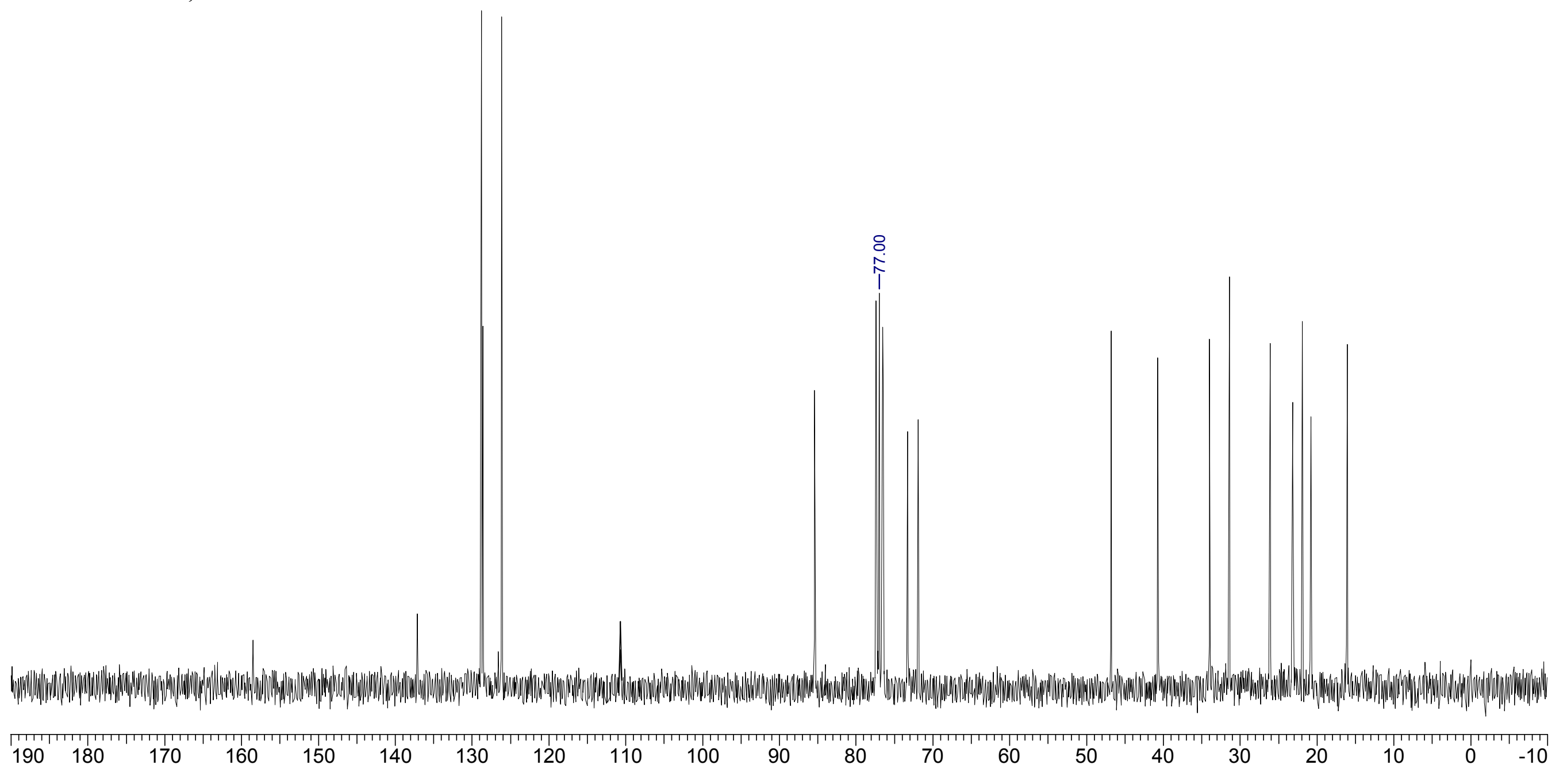




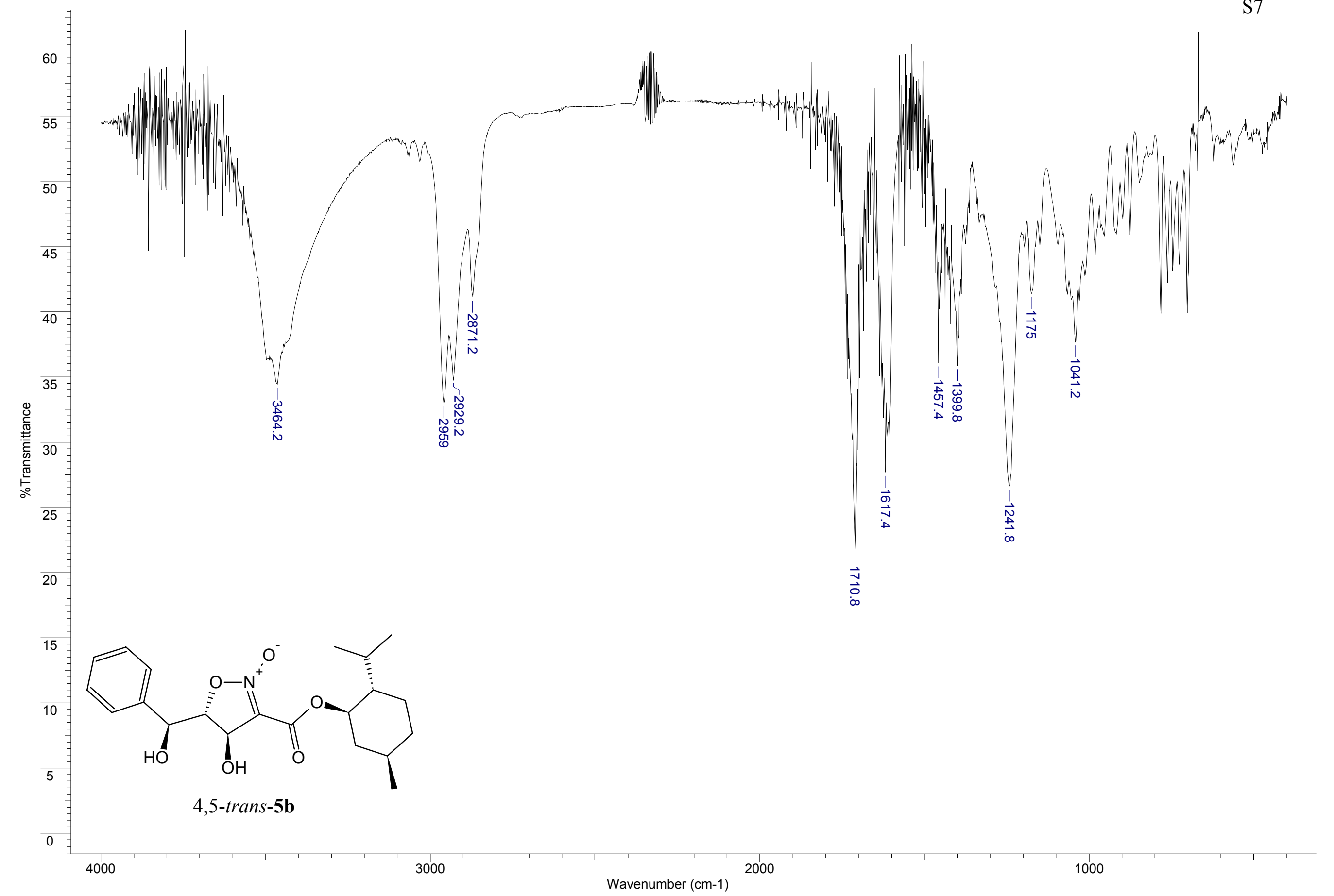




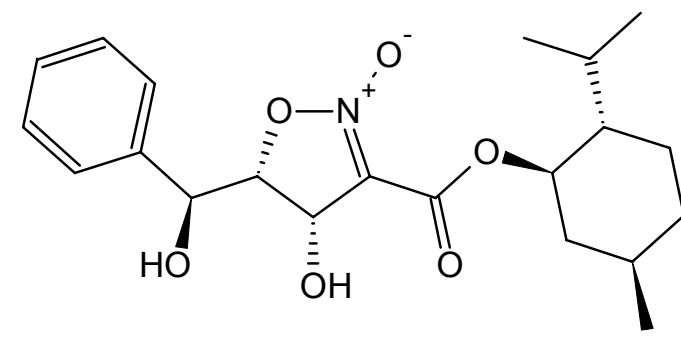

4,5-cis-5b

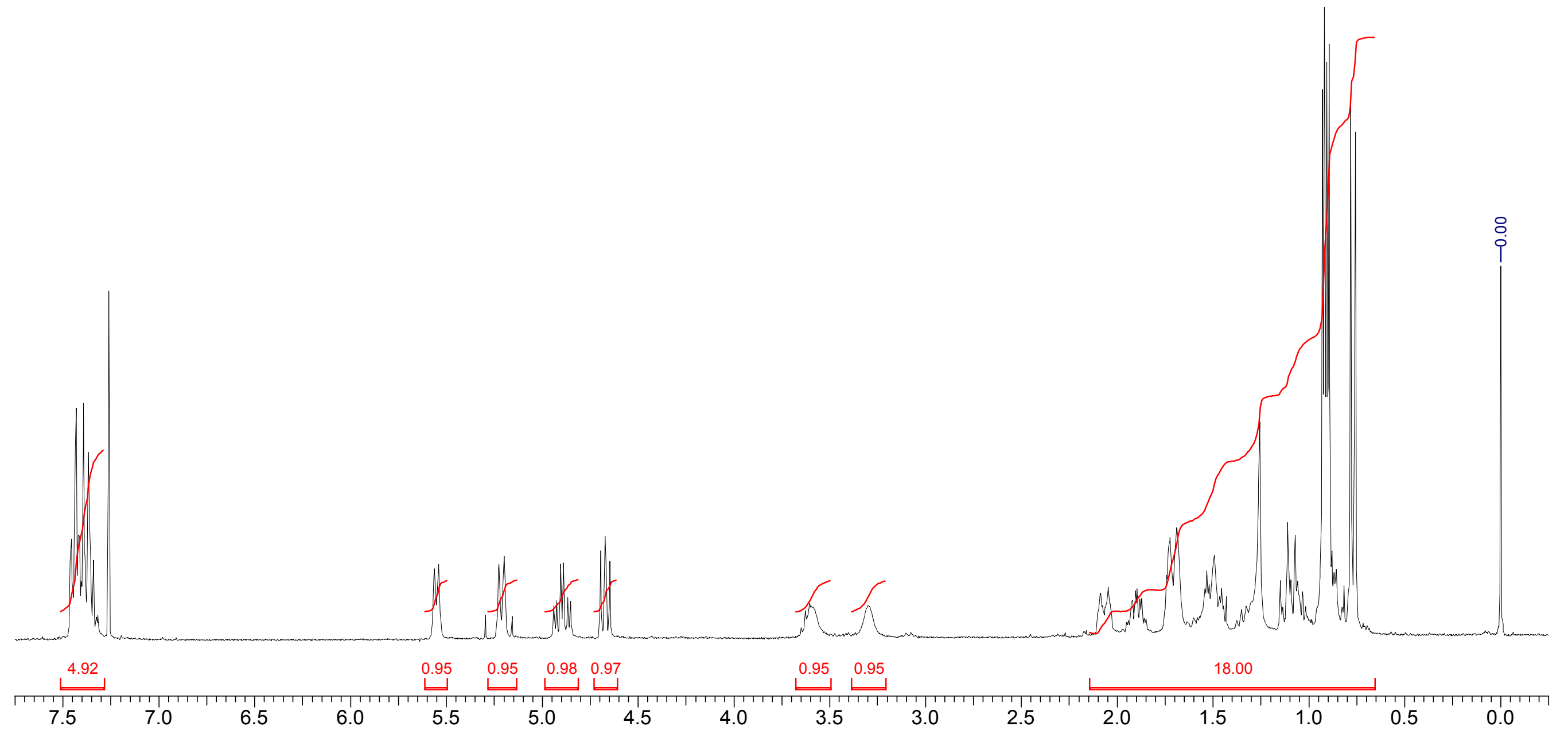




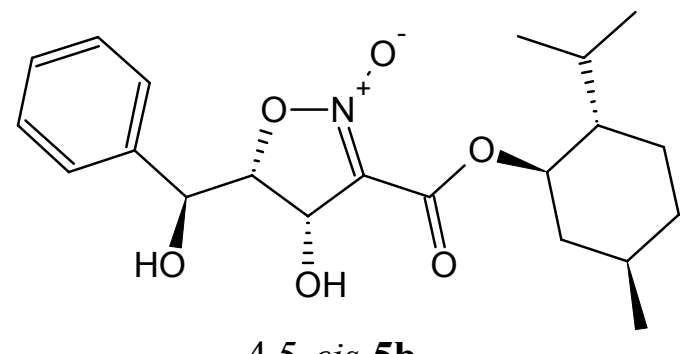

4,5-cis-5b

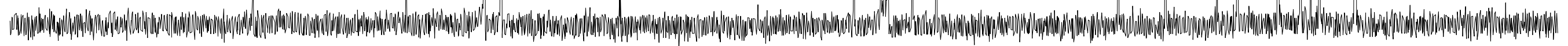

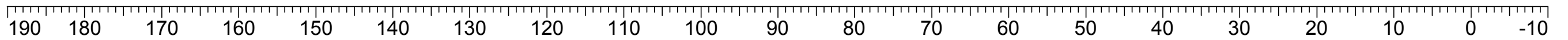




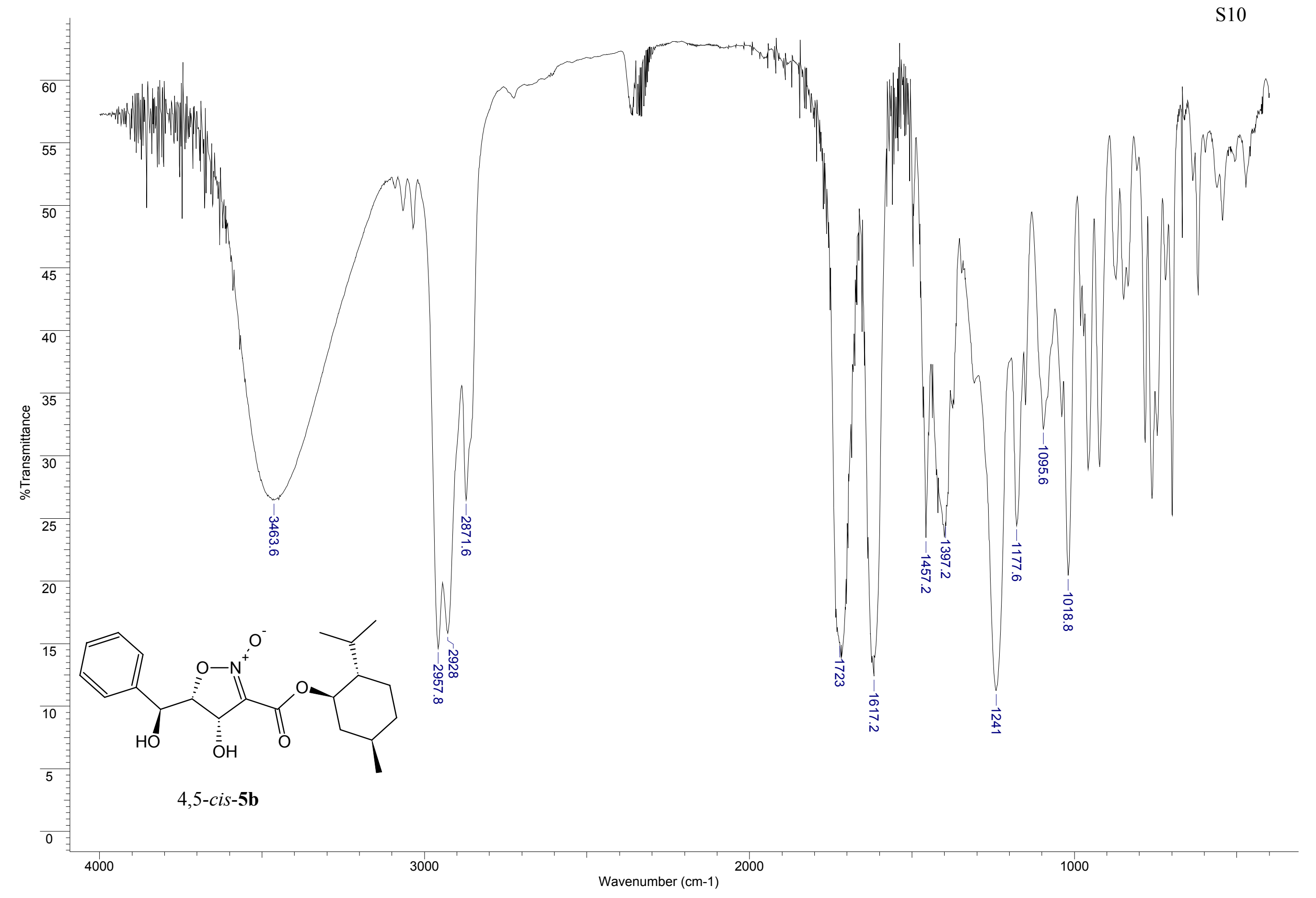




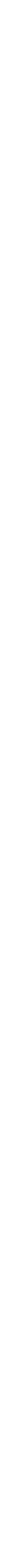




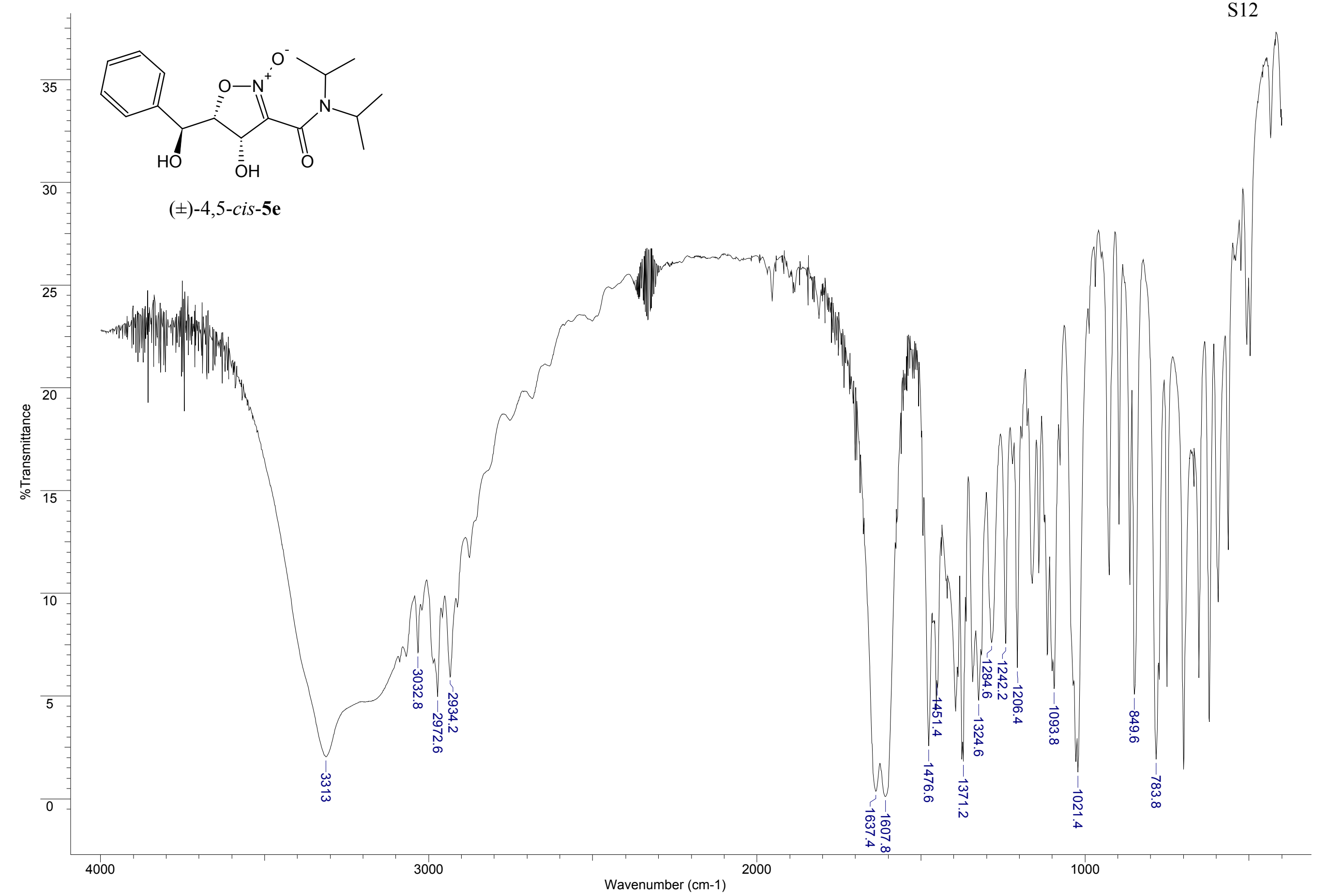




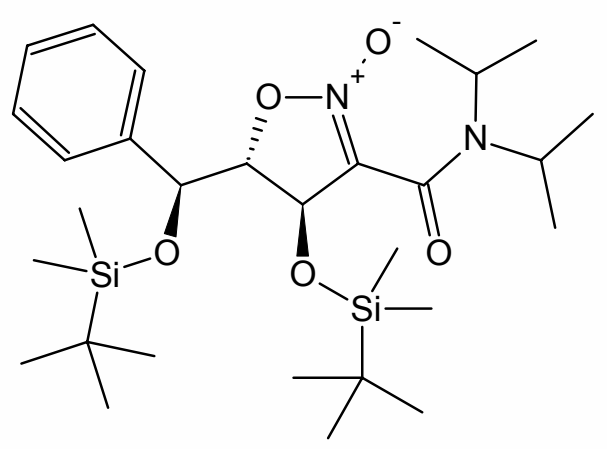

( \pm )-Bis-O-TBDMS-4,5-trans-5e

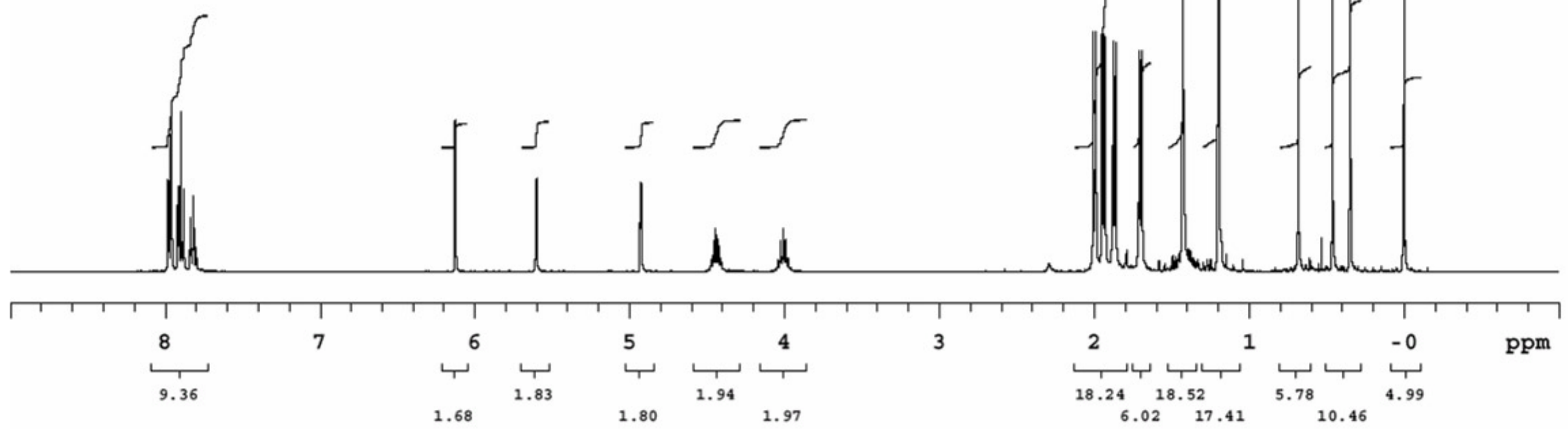




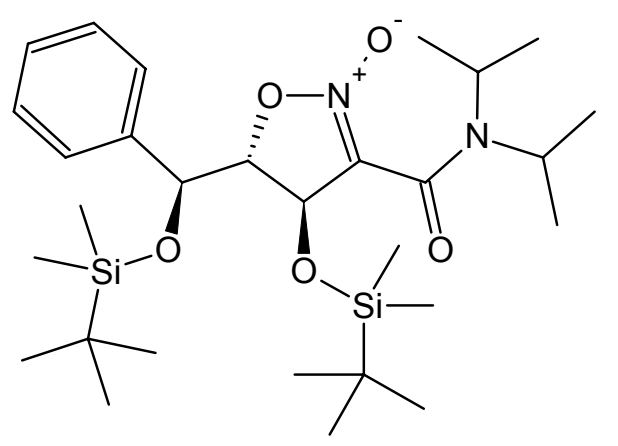

( \pm )-Bis-O-TBDMS-4,5-trans-5e

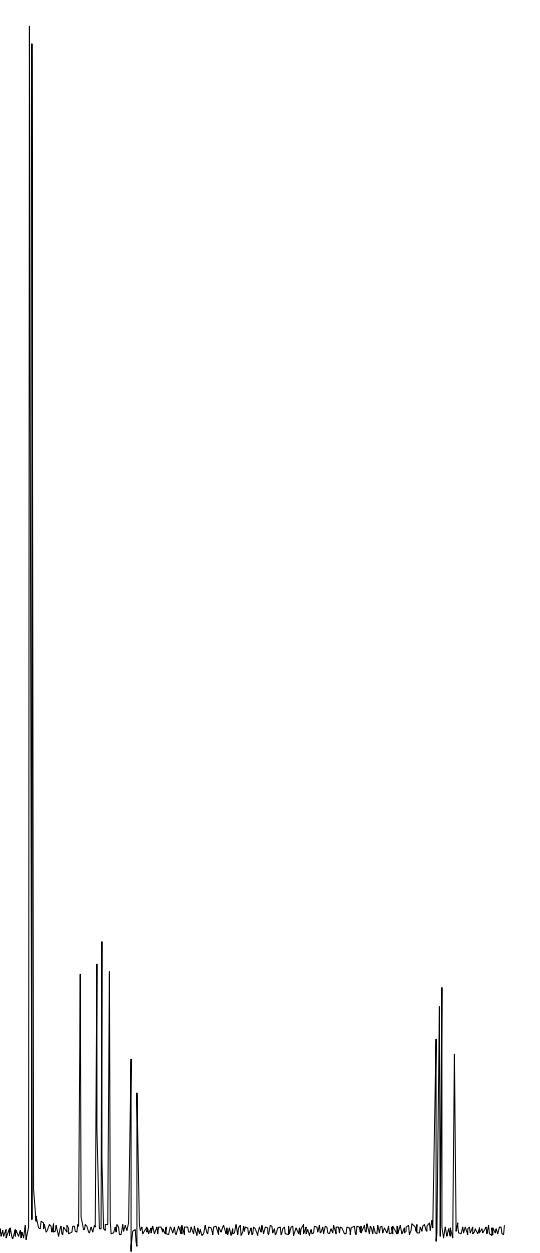

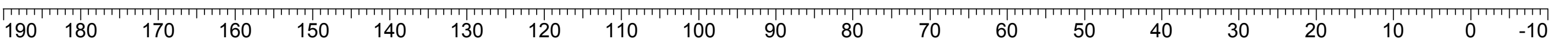
19018

0 


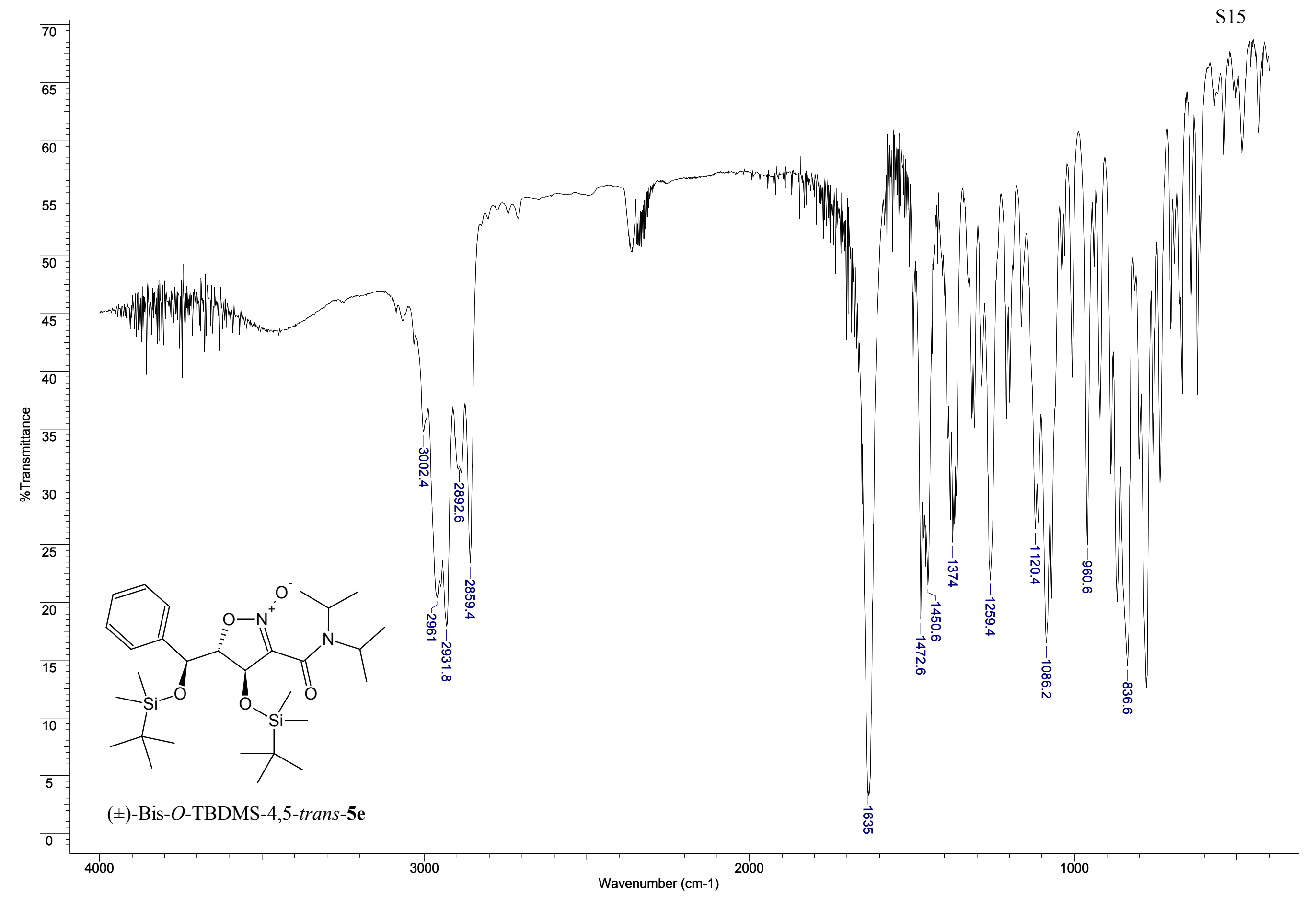




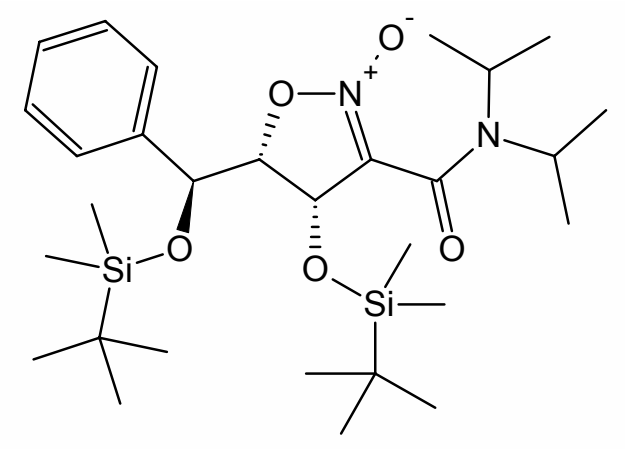

( \pm -Bis- $O$-TBDMS-4,5-cis-5e

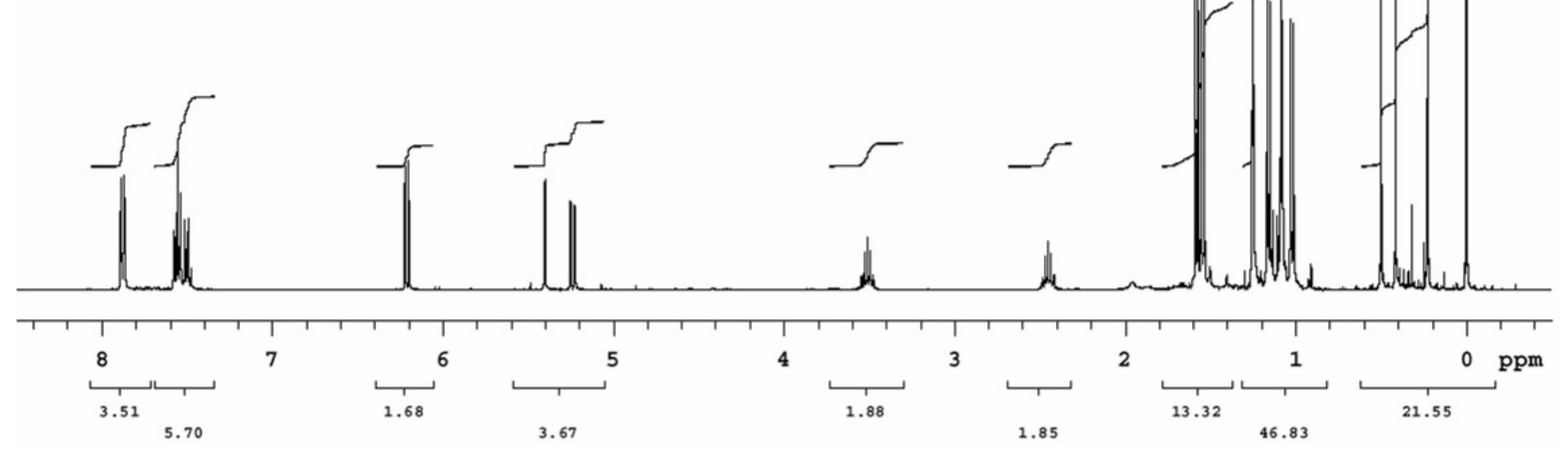




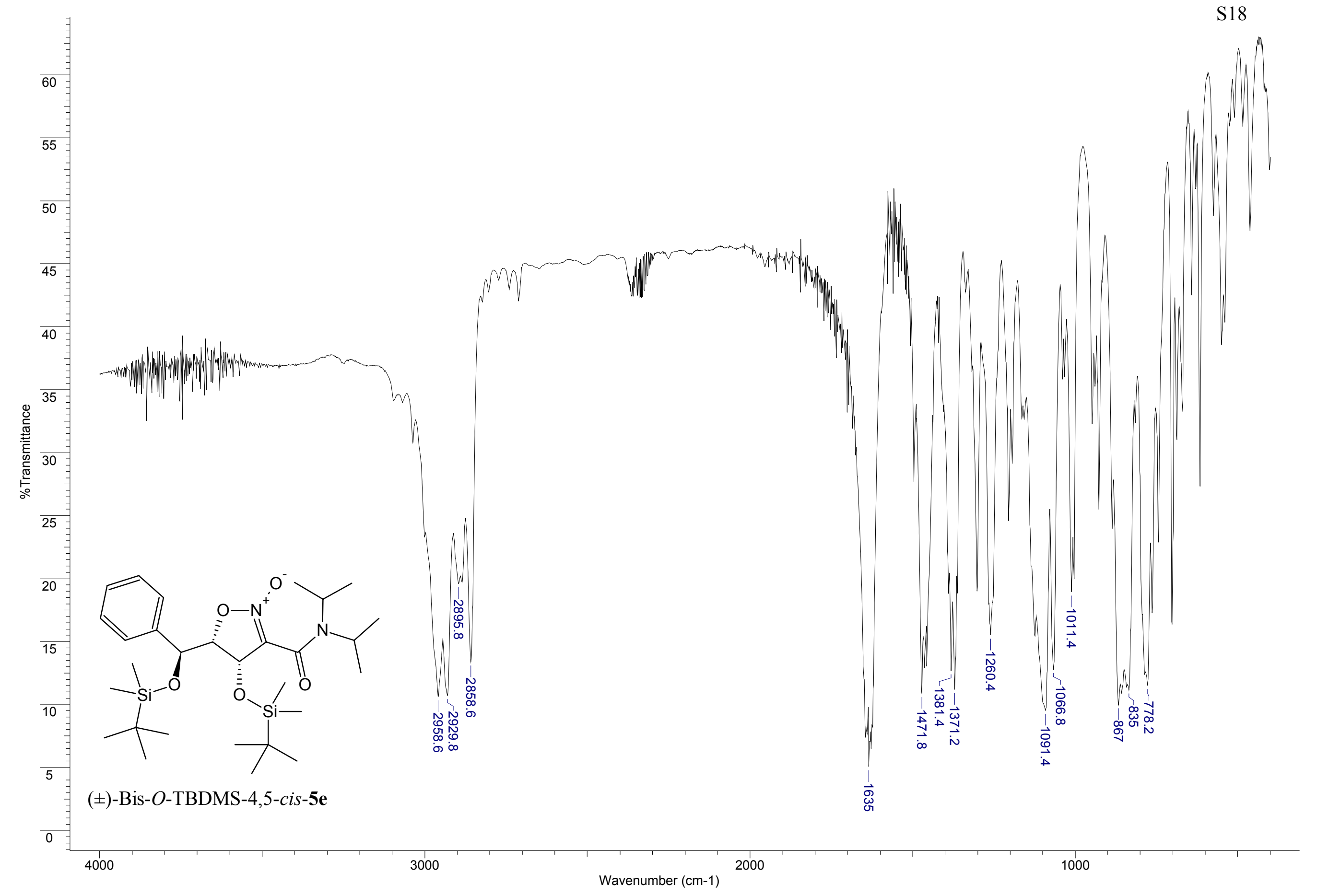




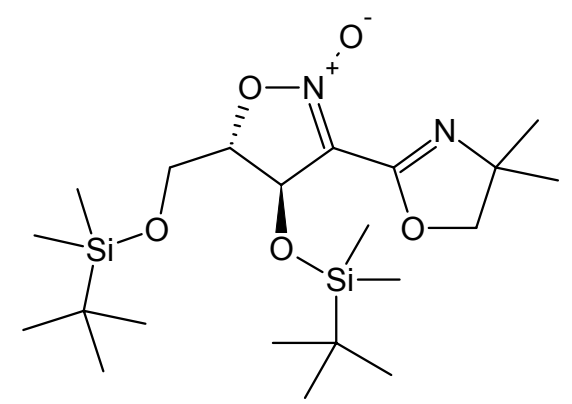

( \pm )-Bis-O-TBDMS-4,5-trans-5f

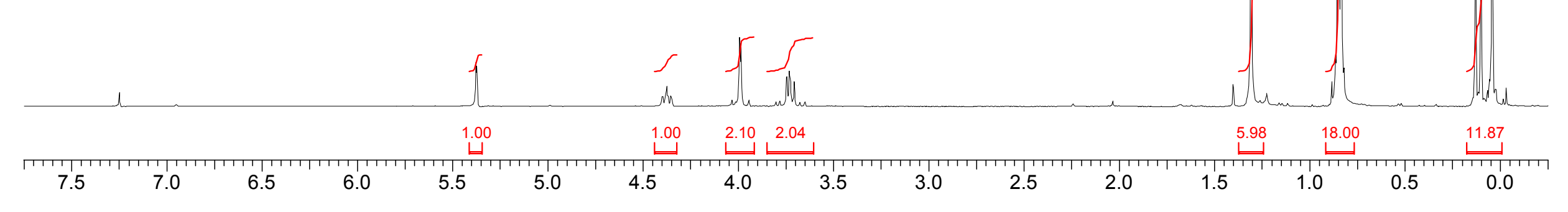




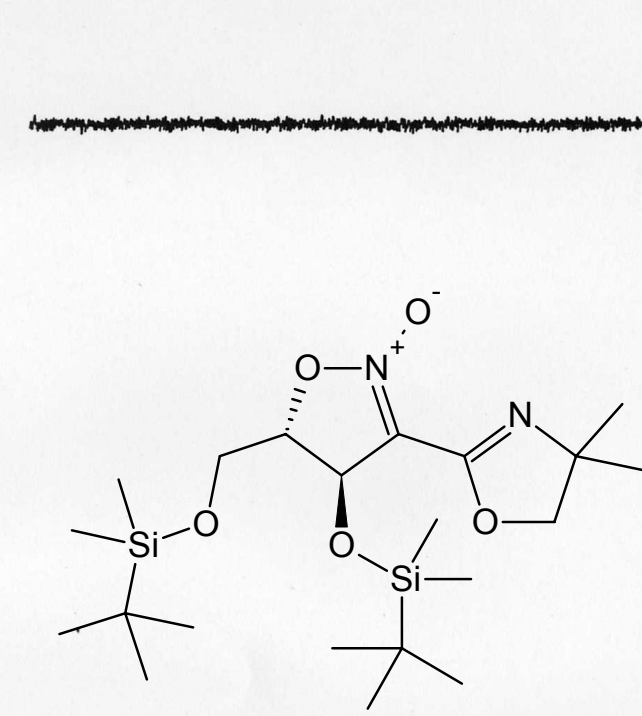

$( \pm)$-Bis- $O$-TBDMS-4,5-trans-5f

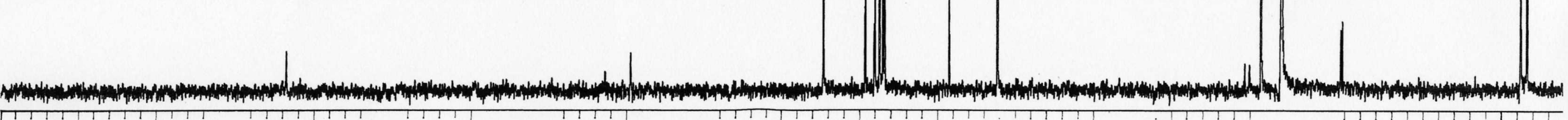




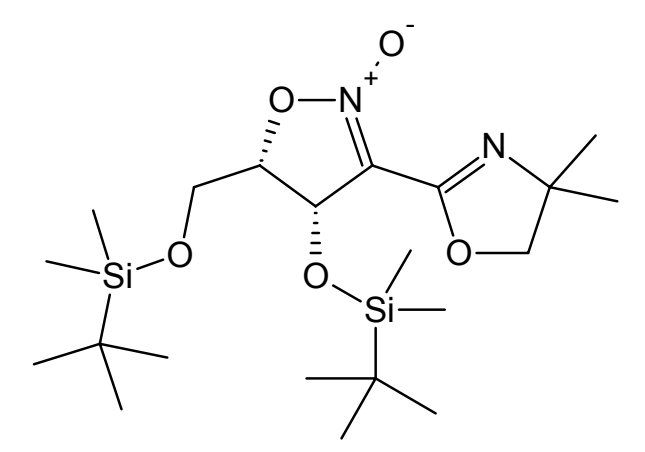

( \pm )-Bis- $O$-TBDMS-4,5-cis-5f

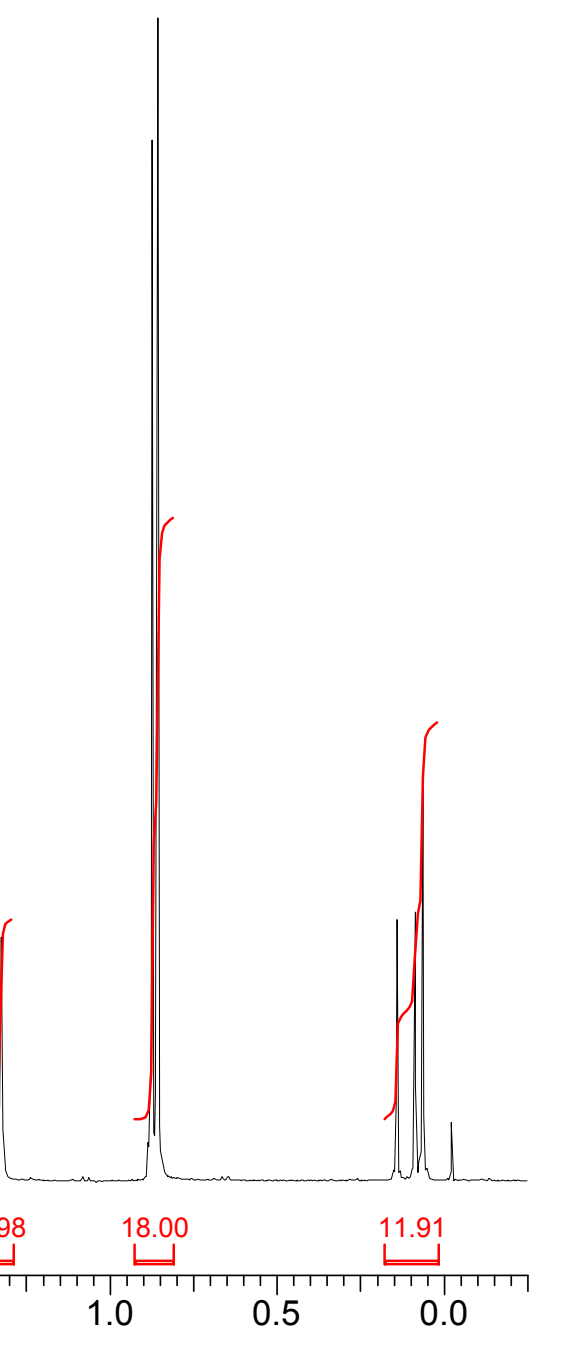




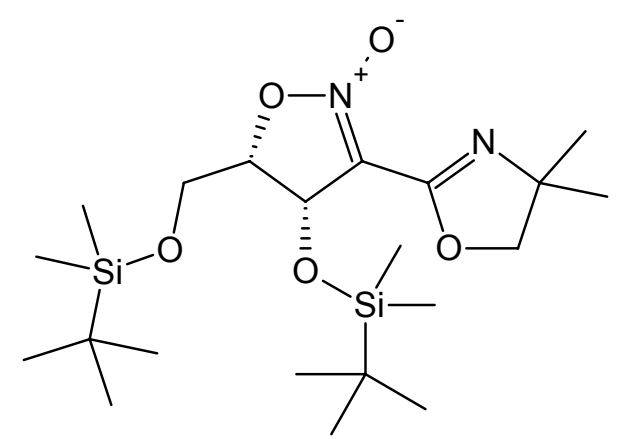

( \pm -Bis- $O$-TBDMS-4,5-cis-5f

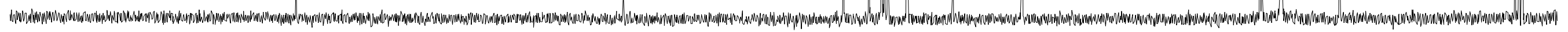

0

